\title{
AVALIAÇÃO DO CATALISADOR Fe/Cu/K/SBA-15 NA SÍNTESE DE FISCHER TROPSCH
}

\author{
R. S. EUARDO ${ }^{1}$, J. C. RODRIGUES ${ }^{1}$, M. G. F. RODRIGUES ${ }^{1}$ \\ ${ }^{1}$ Universidade Federal de Campina Grande, Unidade Acadêmica de Engenharia Química \\ E-mail para contato: raphael_eduardo17@hotmail.com
}

\begin{abstract}
RESUMO - O desenvolvimento de novos materiais, que proporcionem melhores resultados nos processos químicos de transformação e nos custos, é indispensável ao avanço tecnológico do país. Catalisadores a base de ferro possuem grande aplicabilidade no processo de Fischer Tropsch. A utilização de promotores alcalinos potencializa o poder catalítico do ferro suportado. Neste trabalho, o catalisador $\mathrm{Fe} / \mathrm{Cu} / \mathrm{K} / \mathrm{SBA}-15$ foi utilizado na síntese de Fischer Tropsch em reator tipo Slurry Bed. A SBA-15 foi sintetizada pelo método hidrotérmico e os metais impregnados por via úmida. No teste, foram utilizadas as condições de $240^{\circ} \mathrm{C}, 20$ atm, razão $\mathrm{H}_{2} / \mathrm{CO}$ de $1: 1$, no tempo de 6 horas de reação. A SBA-15 e o catalisador foram caracterizados por DRX, EDX e Adsorção de Nitrogênio (Método BET). Os resultados de conversão da reação foram acompanhados por análise de cromatografia gasosa e líquida. O catalisador apresentou área e estrutura hexagonal específicas da SBA-15. O resultado do teste apresentou alta seletividade a metano e moderada seletividade $\mathrm{a}_{5}+$.
\end{abstract}

\section{INTRODUÇÃO}

Um dos fundamentos da sustentabilidade econômica de um país é a sua capacidade de prover logística e energia para o desenvolvimento de sua produção, com segurança e em condições competitivas e ambientalmente sustentáveis (Tolmasquim, 2012). O uso da tecnologia gas to liquid, representada por GTL, pode significar a abertura de novas fronteiras para a utilização do gás natural, agregando valor comercial ao converter gases reagentes em combustíveis líquidos mais valorizados na economia globalizada e em alta demanda por fontes de energia. $\mathrm{O}$ transporte de combustíveis em fase gasosa demanda uma gama de operações que os tornam de difícil utilização quando o consumo se situa a grande distância do centro produtor. Assim, a transformação desses gases combustíveis em combustíveis líquidos, ou seja, o uso da tecnologia GTL, pode baratear e facilitar o transporte do produto gerado (Barros Junior, 2008).

O principal processo Gas To Liquid (GTL), mediante o qual são obtidos produtos líquidos a partir do gás de síntese, é a conversão do gás de síntese em hidrocarbonetos pela síntese de Fischer-Tropsch (STF). Atualmente, existem duas opções bem definidas: o processo STF a baixas temperaturas e a altas temperaturas. O primeiro é empregado na produção de ceras que, após a etapa de hidroprocessamento, são convertidas em nafta ou óleo diesel. O segundo processo é utilizado na produção de gasolina e de alfa-olefinas (Espinoza, 2004).

Os reatores de leito de lama são otimizados para operar em baixa temperatura e produzir altas conversões em graxas com baixas conversões em metano. Dentre as vantagens desse sistema estão o baixo custo, menores pressões de operação, assim como a utilização de uma menor carga de catalisador (Cornils et al., 2013).

Conforme Steynberg e Dry (2004), os catalisadores utilizados no processo STF possuem como fase ativa, metais de transição tais como $\mathrm{Fe}, \mathrm{Ni}$, $\mathrm{Co}$ e $\mathrm{Ru}$, os quais apresentam atividade necessária para sua aplicação comercial. Os catalisadores de ferro para a reação de síntese de Fischer- Tropsch (SFT) são constituídos de ferro sedimentado e promovido por potássio e cobre, visando maior seletividade e atividade, e 


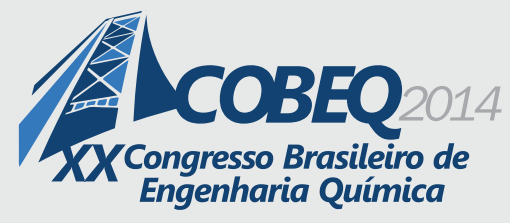

19 a 22 de outubro de 2014

Florianópolis/SC

suportados em alumina $\left(\mathrm{Al}_{2} \mathrm{O}_{3}\right)$ e sílica $\left(\mathrm{SiO}_{2}\right)$, o que confere a esses catalisadores um preço relativamente menor (Zhang et al., 2004). Dois metais estão normalmente presentes nos catalisadores de ferro, o potássio e o cobre. Esses metais devem ser incorporados pela técnica de impregnação ao catalisador de ferro com uma solução aquosa do sal apropriado. O potássio atua na atividade e seletividade do produto do catalisador. $\mathrm{O}$ cobre tem influência significativamente na velocidade com que o catalisador de ferro alcança a máxima atividade, mas há controvérsia acerca do seu impacto em outras propriedades sobre o catalisador (Davis, 2003).

Nos últimos anos tem sido proposta a utilização de catalisadores a base de ferro em fase sólida, podendo ser empregado óxidos de ferro puros ou impregnados em matrizes sólidas (Gonçalves, 2009). A avaliação e a influência do suporte, em relação à área superficial e ao tamanho de poros evidencia que elevadas áreas e estruturas mesoporosas favorecem a reação de SFT, dado o fato de permitirem maior dispersão do metal e acesso dos reagentes e produtos aos poros do catalisador (Griboval-Constanta et al., 2002). A partir da descoberta dos materiais mesoporosos chamados de família M41S, novos sólidos mesoporosos têm sido sintetizados, expandindo significativamente seus potenciais de aplicação em diversos campos, entre eles, a sílica mesoporosa com estrutura hexagonal altamente ordenada chamada de SBA-15, tem se destacado nos últimos tempos (Jung et al., 2004). Diante da necessidade de novas rotas de produção de peneiras moleculares, a cinza da casca de arroz, produto de resíduos sólidos da indústria de geração de energia, mostra-se promissora como subsídio à síntese de materiais mesoporosos. A sílica na casca de arroz é amorfa e transforma-se em sílica cristalina quando aquecida a altas temperaturas (Real, 1996).

Este trabalho tem como objetivo avaliar o desempenho de catalisadores $\mathrm{Fe} / \mathrm{Cu} / \mathrm{K} / \mathrm{SBA}-15$, sintetizados com cinzas da casca de arroz como fonte de sílica, na reação de síntese de Fischer-Tropsch.

\section{MATERIAS E MÉTODOS}

\subsection{Síntese da Peneira molecular SBA-15}

A peneira molecular do tipo SBA-15 foi sintetizada utilizando o direcionador copolímero tribloco Pluronic P123 (polióxido de etileno - polióxido de propileno - polióxido de etileno, $\mathrm{PEO}_{20} \mathrm{PPO}_{70} \mathrm{PEO}_{20}$ ), ácido clorídrico 37\%, água destilada, e fonte de sílica, empregando o método hidrotérmico proposto por Zhao et al. (1998). Estes reagentes foram misturados para obter um hidrogel reativo de composição molar 1.0 Fonte de Sílica: 0,017 P123: 5,7 HCl: $193 \mathrm{H}_{2} \mathrm{O}$. O direcionador $\mathrm{P} 123$ foi dissolvido em água destilada e ácido clorídrico, com agitação e na temperatura de $35^{\circ} \mathrm{C}$. Após homogeneização, adicionou-se as cinzas da casca de arroz como fonte de sílica, obtidas pelo tratamento térmico da casca de arroz (4 horas em forno mufla a $600^{\circ} \mathrm{C}$ ), submetidas após o tratamento térmico a um tratamento ácido (ácido clorídrico de concentração $1 \mathrm{M}$, temperatura de $100^{\circ} \mathrm{C}$ sob agitação, em um tempo de contato de 2 horas e razão de cinzas de casca arroz/volume de solução de $25 \mathrm{~g} / 500 \mathrm{ml}$ ). A mistura foi mantida sob agitação, na temperatura de $35^{\circ} \mathrm{C}$ por 24 horas $(\mathrm{pH}=1)$, sendo obtido um gel homogêneo, o qual foi transferido para cadinhos de teflon, acondicionados em autoclaves de aço inox. As autoclaves foram postas em estufa previamente aquecida a $100^{\circ} \mathrm{C}$, permanecendo nesta temperatura por 48 horas, seguindo metodologia proposta por Rodrigues (2012). $\mathrm{O}$ material obtido foi calcinado em forno mufla sob taxa de aquecimento de $10^{\circ} \mathrm{C} / \mathrm{min}$, até $550^{\circ} \mathrm{C}$, permanecendo nesta temperatura por 4 horas.

\subsection{Preparação do catalisador $\mathrm{Fe} / \mathrm{Cu} / \mathrm{K} / \mathrm{SBA}-15$}

$\mathrm{O}$ catalisadores $\mathrm{Fe} / \mathrm{Cu} / \mathrm{K} / \mathrm{SBA}-15$ foi preparado, adotando-se o método de impregnação dos metais por via úmida. Os catalisadores foram preparados em base molar de $100 \mathrm{Fe} / 5 \mathrm{Cu} / 18 \mathrm{~K} / 139 \mathrm{SiO}_{2}$, utilizando soluções de nitrato de ferro nonahidratado $\mathrm{Fe}\left(\mathrm{NO}_{3}\right)_{3} .9 \mathrm{H}_{2} \mathrm{O}$, nitrato de cobre trihidratado $\mathrm{Cu}\left(\mathrm{NO}_{3}\right)_{2} \cdot 3 \mathrm{H}_{2} \mathrm{O}$ e bicarbonato de potássio $\mathrm{KHCO}_{3}$, como fontes metálicas e SBA-15 como fonte de sílica. A SBA-15 foi posta em contato com 


\section{9 a 22 de outubro de 2014 \\ Florianópolis/SC}

as soluções dos metais sob agitação a temperatura ambiente e após 30 minutos, a mistura foi acondicionada em estufa a $60^{\circ} \mathrm{C}$ durante 24 horas para secagem. O material foi calcinado sob atmosfera de nitrogênio segundo um procedimento em três estágios. Inicialmente a amostra foi submetida, a partir da temperatura ambiente, a uma taxa de aquecimento de $5^{\circ} \mathrm{Cmin}^{-1}$ até $100^{\circ} \mathrm{C}$, permanecendo neste patamar por $10 \mathrm{~min}$, seguida por uma nova taxa de aquecimento de $10^{\circ} \mathrm{Cmin}^{-1}$ até atingir $300^{\circ} \mathrm{C}$. $\mathrm{O}$ catalisador permaneceu nessa temperatura durante $5 \mathrm{~h}$. Com este procedimento de calcinação, ocorreu a decomposição dos os íons nitratos, sendo convertidos em óxidos.

\subsection{Síntese de Fischer-Tropsch}

As reações de síntese de Fischer-Tropsch foram realizadas em um reator de leito de lama (modelo PARR) envolvido em uma manta térmica, alimentado com $3 \mathrm{~g}$ de catalisador Fe/Cu/K/SBA-15 e $150 \mathrm{ml}$ de hexadecano, utilizado com fase líquida inerte. Em seguida, foram adicionados os gases $\mathrm{CO}$ e $\mathrm{H}_{2}$ nas proporções de 1:1, em fluxo gasoso em batelada, alimentado através de controladores de fluxo mássico. O sistema de agitação do reator é de acoplamento magnético, permitindo a recirculação da fase gás através do leito de lama, ajustado para $760 \mathrm{rpm}$. Os catalisadores foram ativados in situ. Depois de alcançada a temperatura de $240^{\circ} \mathrm{C}$ e pressão de 20 atm, a síntese transcorreu por um período de 6 horas.

\subsection{Caracterização}

\subsubsection{Difração de Raios X (DRX)}

Os dados coletados das amostras foram analisados utilizando o método do pó empregando-se um difratômetro Shimadzu XRD-6000 com radiação $\mathrm{CuK \alpha}$, tensão de $40 \mathrm{KV}$, corrente de $30 \mathrm{~mA}$, tamanho do passo de $0,0202 \theta$ e tempo por passo de 1,000 s, com velocidade de varredura de $2^{\circ}(2 \theta) / \mathrm{min}$, com ângulo $2 \theta$ percorrido de $0,5^{\circ}$ a $8^{\circ}$ e 35 a $75^{\circ}$.

\subsubsection{Análise Química através do Espectrômetro de Raios X por Energia Dispersiva (EDX)}

As composições das amostras foram analisadas em um espectrômetro EDX-700 Shimadzu.

\subsubsection{Adsorção Física de Nitrogênio (Método de BET)}

$\mathrm{Na}$ obtenção das isotermas de adsorção e dessorção de nitrogênio a $-196^{\circ} \mathrm{C}$ utilizou-se um aparelho ASAP 2020 da Micromeritics. Para isso $100 \mathrm{mg}$ de cada amostra foi previamente tratada nas as seguintes condições: Fase de vacou: Taxa de aquecimento: $10^{\circ} \mathrm{C} / \mathrm{min}$, Temperatura: $90^{\circ} \mathrm{C}$, Vácuo setpoint: 10 micronHg, Tempo de vácuo: $60 \mathrm{~min}$. Fase de aquecimento: Taxa de aquecimento: $10^{\circ} \mathrm{C} / \mathrm{min}$ e Temperatura de $350^{\circ} \mathrm{C}$.

\subsubsection{Cromatografia Gasosa}

Para análise dos produtos da reação de Fischer-Tropsch foi utilizado um cromatógrafo a gás (Thermos Ultra). Amostras da fase líquida foram retiradas após resfriamento do reator e também analisadas por cromatografia. A análise cromatográfica utilizou uma coluna de detectores de condutividade térmica (TCD) e outra de ionização em chama (FID). 


\section{RESULTADOS E DISCUSSÕES}

Na Figura 1 está apresentado o difratograma de raios-x da peneira mesoporosa sintetizada (a), e calcinada (b), apresentando a estrutura hexagonal ordenada característica da SBA-15, semelhante aos padrões observados na literatura $(1,12)$. O pico principal de difração, cujo índice de Miller é $(100)$, referente aos planos cristalinos, característico desse tipo de material, pode ser observado.

a)

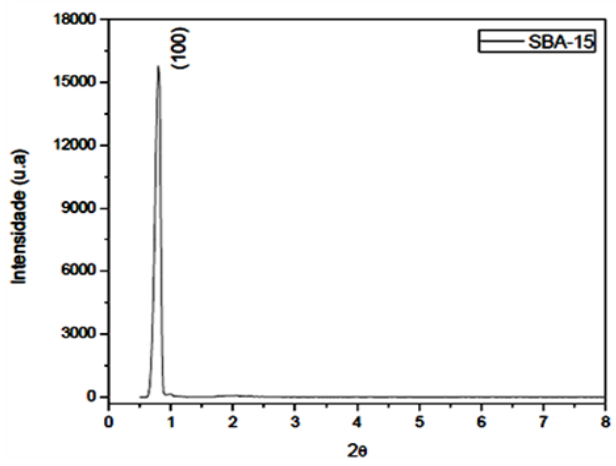

b)

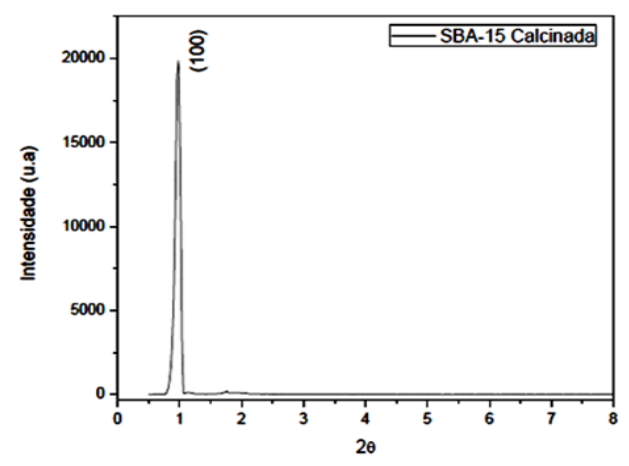

Figura 1 - Difratogramas de raios x da peneira molecular SBA-15: a) Sintetizada e b) Calcinada.

Devido a retirada do direcionado, ocorreu uma reorganização das ligações presentes na estrutura (formação de novas ligações siloxanas ( $\mathrm{Si}-\mathrm{O}-\mathrm{Si})$ e condensação das ligações silanois ( $\equiv \mathrm{Si}-\mathrm{OH})$, convergindo a um aumento no ordenamento do sistema poroso, observado pelo aumento da intensidade do pico característico, ocorrendo uma contração do parâmetro de rede hexagonal (Yu et al., 2000). Na Tabela 1 estão apresentados os valores do parâmetro mesoporoso $\mathrm{a}_{0}$ e dos ângulos $2 \theta$, com suas respectivas distâncias interplanares no plano (hkl) para as amostras SBA- 15.

Tabela 1 - Valores dos ângulos $2 \theta$, suas respectivas distâncias interplanares e o parâmetro mesoporoso $\mathrm{a}_{0}$ para o plano $\left(\begin{array}{lll}1 & 0 & 0\end{array}\right)$ da amostra de SBA-15 e do catalisador.

\begin{tabular}{|c|c|c|c|c|}
\hline Amostra & $2 \theta$ & $\mathrm{hkl}$ & $\mathrm{d}_{\text {(hkl) }}(\AA)$ & $\mathrm{a}_{0}(\mathrm{~nm})$ \\
\hline SBA-15 (sintetizada) & 0,84 & 100 & 104,93 & 12,1 \\
\hline SBA-15 (calcinada) & 0,94 & 100 & 93.980 & 10,8 \\
\hline
\end{tabular}

Na Figura 2 são apresentadas as isotermas de adsorção-dessorção de $\mathrm{N}_{2}$ a $-196^{\circ} \mathrm{C}$ referentes à amostra de SBA-15 calcinada.

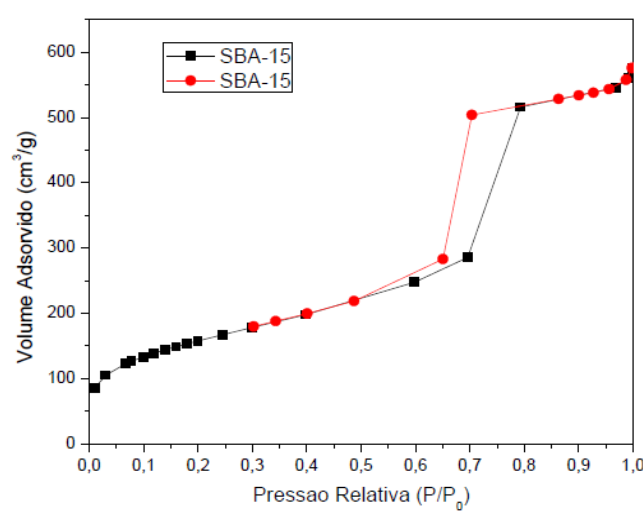


Figura 2 - Isotermas de adsorção-dessorção de $\mathrm{N}_{2}$ a $-196^{\circ} \mathrm{C}$ da amostra de SBA-15 calcinada.

Verificou-se por meio das análises de adsorção-dessorção de N2 isotermas do tipo IV e histerese do tipo $\mathrm{H} 1$, constituída por dois ramos verticais durante um intervalo de 0,6 a $0,8\left(\mathrm{P} / \mathrm{P}_{0}\right)$ no eixo das abscissas. Esse comportamento é característico de materiais mesoporosos do qual a SBA-15 faz parte.

$\mathrm{O}$ difratograma do catalisador $\mathrm{Fe} / \mathrm{Cu} / \mathrm{K} / \mathrm{SBA}-15$ está apresentado na Figura 3.

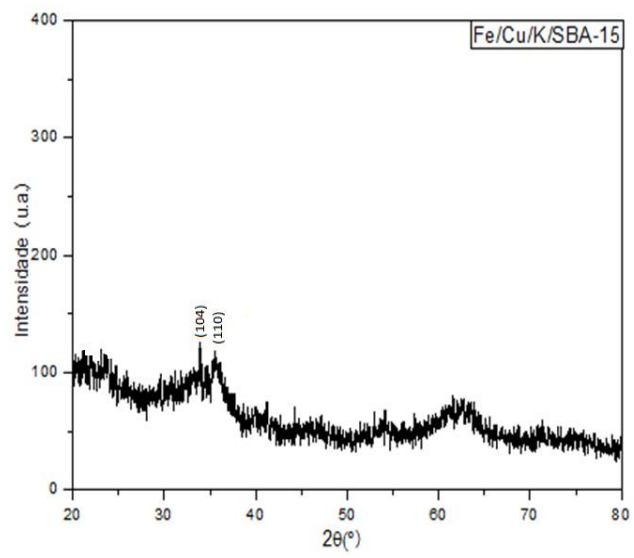

Figura 3 - Difratograma de raios x do catalisador Fe/Cu/K/SBA-15 após impregnação e calcinação.

Pela análise do difratograma, o ferro deve estar bem disperso na estrutura da SBA-15, o que favorece a estabilidade térmica do catalisador (Wang et al., 2005). Os picos de Ferro ou $\mathrm{Fe}_{2} \mathrm{O}_{3}$ (na forma de hematita ou maghemita) não foram detectados, o que indica que as nanopartículas do metal ou do óxido estão bem dispersas no suporte. Tal fato evidencia o baixo grau de cristalinidade do catalisador e os pequenos tamanhos de partículas do metal na estrutura, caracterizando-se como um material com nanopartículas de ferro disperso e semicristalino.

Os resultados de composição química estão descritos na Tabela 2.

Tabela 2 - Composição química das amostras de SBA-15 e do catalisador.

\begin{tabular}{|c|c|c|c|c|c|}
\hline Amostra & $\mathrm{SiO}_{2}(\%)$ & $\mathrm{Fe}_{2} \mathrm{O}_{3}(\%)$ & $\mathrm{CuO}(\%)$ & $\mathrm{K}_{2} \mathrm{O}(\%)$ & Outros \\
\hline SBA-15 (sintetizada) & 99,3 & - & - & - & 0,7 \\
\hline SBA-15 (calcinada) & 99,5 & - & - & - & 0,5 \\
\hline $\mathrm{Fe} / \mathrm{Cu} / \mathrm{K} / \mathrm{SBA}-15$ & 41,0 & 51,3 & 2,7 & 4,7 & 0,3 \\
\hline
\end{tabular}

Verificou-se que as amostras de SBA-15 apresentaram alto teor de sílica $\left(\mathrm{SiO}_{2}\right)$, previstos para esse tipo de material. Para o catalisador $\mathrm{Fe} / \mathrm{Cu} / \mathrm{K} / \mathrm{SBA}-15$, os teores de óxido de ferro, óxido de cobre e óxido de potássio foram $51,3 \%, 2,7 \%$ e $4,7 \%$, respectivamente. Na forma de metal, os valores obtidos de $35,87 \%, 2,16 \%$ e 3,9\% de ferro, cobre e potássio, respectivamente, indiciam convergência com os valores pré-definidos para base molar de $100 \mathrm{Fe} / 5 \mathrm{Cu} / 18 \mathrm{~K} / 139 \mathrm{SiO}_{2}$, indicando a eficiência do processo de dispersão física dos metais na estrutura mesoporosa da SBA-15.

Na Tabela 3 são apresentados os Valores de conversão e seletividade a hidrocarbonetos da reação de Ficsher-Tropsch utilizando o catalisador Fe/Cu/K/SBA-15.

Tabela 3 - Valores de conversão e seletividade a hidrocarbonetos da reação de Ficsher-Tropsch utilizando o catalisador Fe/Cu/K/SBA-15.

\begin{tabular}{|l|l|l|l|l|l|l|}
\hline Catalisador & $\mathrm{C}_{1}$ & $\mathrm{C}_{2}$ & $\mathrm{C}_{3}$ & $\mathrm{C}_{4}$ & $\mathrm{C}_{5}+$ & Conversão \\
\hline
\end{tabular}




\begin{tabular}{|c|c|c|c|c|c|c|}
\hline $\mathrm{Fe} / \mathrm{Cu} / \mathrm{K} / \mathrm{SBA}-15$ & 59,2 & 2,3 & 3,1 & 13,9 & 21,5 & $30 \%$ \\
\hline
\end{tabular}

Analisando os dados da Tabela 3, dentro das condições de reação estudada, verificou-se que o catalisador $\mathrm{Fe} / \mathrm{Cu} / \mathrm{K} / \mathrm{SBA}-15$ apresentou baixa seletividade para hidrocarbonetos líquidos $\mathrm{C}_{5+}$ bem como moderada conversão do gás de síntese, sendo necessários ajustes nas condições de processo (tempo, temperatura, pressão e razão de gás) para um melhor desempenho do catalisador na síntese.

$\mathrm{Na}$ figura 4, é apresentado o difratograma de raios x das amostra de catalisador utilizado no teste de síntese de Fischer-Tropsch.

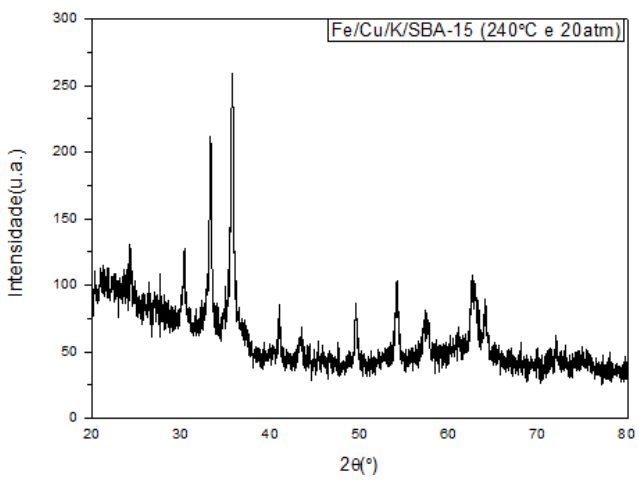

Figura 4 - Difratograma de raios $\mathrm{x}$ das amostras do catalisador $\mathrm{Fe} / \mathrm{Cu} / \mathrm{K} / \mathrm{SBA}-15$ após utilização na síntese de Fischer-Tropsch nas condições de $240^{\circ} \mathrm{C}$ e 20 atm.

Pode-se observar pela Figura 4 que o catalisador apresentou picos na faixa de $33^{\circ}, 36^{\circ}, 50^{\circ}$ e $54^{\circ}$, na faixa de $2 \theta$, característico da fase $\alpha-\mathrm{Fe} 2 \mathrm{O} 3$ (Hematita). O surgimento desses picos é favorecido pelo alto teor de potássio nas amostras do catalisador utilizado, tornando maior a intensidade dos picos, respaldado por estudos (Jin e Datye, 2000). O pico na angulação em torno de $58^{\circ}$ é referente à fase $\gamma$-Fe2O3 (Maghemita). Tal fato se deve a formação de estruturas cristalinas que são formadas pelas presenças dos promotores estruturais presentes.

Verifica-se a alta cristalinidade do catalisador pós-síntese de Fischer-Tropsch. O catalisador anterior à síntese apresentou baixa cristalinidade, o que remete a baixa presença dos óxidos metálicos, ideal para a reação de FTS por favorecer a ativação para a dissociação do monóxido de carbono e a formação de hidrocarbonetos. A reutilização dos catalisador poderia favorecer a reação de Water Gas Shift, devido a presença dos óxidos metálicos, necessitando a redução das fases óxidas para desempenho semelhante ao catalisador sintetizado.

\section{CONCLUSÕES}

Neste trabalho, cinzas da casca de arroz amorfa foram utilizadas com fonte de sílica para síntese da peneira molecular SBA-15. Os resultados obtidos mostraram que foi possível sintetizar a peneira molecular SBA-15 utilizando a cinza da casca de arroz como fonte de sílica, e que suas características se aproximam (ou se assemelham) da peneira molecular SBA-15 utilizando fontes convencionais na sua preparação.

Pela análise de composição química, o catalisador $\mathrm{Fe} / \mathrm{Cu} / \mathrm{K} / \mathrm{SBA}-15$ apresentou teores de metal na forma de óxidos bem próximos dos valores pré-definidos. A dispersão do ferro na estrutura da peneira molecular foi bem sucedida, analisada pelo difratograma de Raios X do catalisador em questão, apresentando semicristalinidade e nanopartículas de metais. A ausência dos picos característicos da hematita e da maghemita, mesmo com a presença do ferro na estrutura do catalisador comprovada pela análise química por espectro de raios X por energia dispersiva, caracterizam a boa dispersão do metal na estrutura da SBA-15. 
$\mathrm{O}$ catalisador $\mathrm{Fe} / \mathrm{Cu} / \mathrm{K} / \mathrm{SBA}-15$ apresentou alta seletividade para metano e baixa seletividade para hidrocarbonetos líquidos $\mathrm{C}_{5}$ + bem como moderada conversão do gás de síntese nas condições de $240^{\circ} \mathrm{C}, 20$ atm e 6 horas de reação.

As análises pelo difratograma de Raios $\mathrm{X}$ das amostras utilizadas na síntese de Fischer-Tropsch indicaram que os materiais apresentaram altos picos na faixa de $33^{\circ}, 36^{\circ}, 50^{\circ}$ e $54^{\circ}$, na faixa de $2 \theta$, característicos da fase $\alpha-\mathrm{Fe}_{2} \mathrm{O}_{3}$ (Hematita), com intensidade crescente com a elevação nas condições de processo, apresentando alta cristalinidade das suas estruturas.

\section{REFERÊNCIAS}

JIN, Y.; DATYE, A. K. Phase transformations in iron Fischer-Tropsch catalysts during temperatureprogrammed reduction. Journal of Catalysis V.196, p.8-17, 2000.

WANG, X.Q; GE, H.L; JIN, H.X; CUI, Y.J. Influence of Fe on the thermal stability and catalysis of SBA-15 mesoporous molecular sieves. Microporous and Mesoporous Materials. Vol.86, p.335-340, 2005.

YU, J; SHI, J. L.; WANG, L. Z.; RUAN, M. L.; YAN D. S. Room temperature synthesis of mesoporous aluminosilicate materials Ceram. Int., V.26, p.359-362, 2000.

ZHAO, D., HUO, Q., FENG, J., B.F. CHMELKA. G.D. Stucky, Nonionic Triblock and Star Diblock Copolymer and Surfactant Syntheses of Highly Ordered, Hydrothermally Stable, Mesoporous Silica Structures. Journal of the American Chemical Society. v.120, p.6024-6036, 1998.

RODRIGUES, J. J.; PECCHI, G.; FERNANDES, F. A. N.; RODRIGUES, M. G. F. Ruthenium promotion of Co/SBA-15 catalysts for Fischer-Tropsch synthesis in slurry-phase reactors. Journal of Natural Gas Chemistry, Vv.21, p. 722-728, 2012.

TOLMASQUIM, M. Ti. Perspectivas e planejamento do setor energético no Brasil. Estud. av., São Paulo, v. 26, n. 74, p.247-260, 2012.

BARROS JUNIOR, A. F. Modelagem e Simulação da Síntese Fischer-Tropsch em Reator Tubular de Leito Fixo com Catalisador de Ferro. Dissertação (mestrado) - Universidade Federal do Ceará, Programa de PósGraduação em Engenharia Química, Fortaleza, p.106, 2008.

ESPINOZA, R. L.; STEYNBERG, A. P.; JAGER, B.; VOSLOO, A. C. Low temperature Fischer-Tropsch synthesis from a Sasol perspective. Applied Catalysis A: General, v.186, p.13- 26, 2004.

CORNILS, Boy; HERRMANN, Wolfgang A.; WONG, Chi-Huey; ZANTHOFF, Horst Werner. Catalysis from A to Z: a concise encyclopedia. 4 ed. Alemanha: Wiley-VCH Verlag GmbH \& Co. KGaA, 2013.

STEYNBERG, A.; DRY, M. Fischer-Tropsch Technology. Studies in surface science and catalysis, v.154, 2004.

ZHANG, Y.; SHINODA, M.; TSUBAKI, N. Development of bimodal cobalt catalysts for Fischer-Tropsch synthesis. Catalysis today, v. 93, p.55-63, 2004.

DAVIS, B.H. Fischer-Tropsch synthesis: relationship between iron catalyst composition and process variables. Catalysis Today, v.84, p.83-98, 2003. 
GONÇALVES, M.; OLIVEIRA, L. C. A.; GUERREIRO, M. C. Síntese e Caracterização de Nanopartículas de Óxido de Ferro Suportadas em Matriz Carbonácea: Remoção do Corante Orgânico Azul de Metileno em Água. Química Nova, V.32, No. 7, p.1723-1726, 2009.

GRIBOVAL-CONSTANTA, A.; KHODAKOV, A. Y.; BECHARA, R.; ZHOLOBENKO, V. L. Support mesoporosity: a tool for better control of catalytic behavior of cobalt supported Fischer Tropsch catalysts. Studies in Surface Science and Catalysis, v. 144, p. 609-616, 2002.

JUNG, J. S.; CHOI, K. H.; JUNG, Y. K.; LEE, S. H.; GOLUB, V. O.; MALKINSKI L.; O'CONNOR, C. J. Preparation and characterization of $\mathrm{Fe} 2 \mathrm{O} 3$ nanoparticles in SBA-15 host material. Journal of Magnetism and Magnetic Materials, v. 272, p.1157-1159, 2004.

REAL, C; ALCALA, M.; CRIADO, J. Preparation of Silica from Rice Husks. Journal of the American Ceramic Society. v.79, p.2012-2016, 1996. 